\title{
Effects of Lamellolysin From a Parasitoid Wasp on Drosophila Blood Cells In Vitro
}

\author{
R.M. RIZKI AND T.M. RIZKI \\ Department of Biology, The Üniversity of Michigan, \\ Ann Arbor, Michigan 48109
}

\begin{abstract}
Female parasitoid Leptopilina heterotoma inject a factor, lamellolysin, along with their eggs into the host hemocoel to destroy selectively host hemocytes that encapsulate foreign objects. In parasitized Drosophila melanogaster larvae, these hemocytes (lamellocytes) change from discoidal cells to bipolar cells that no longer adhere to each other to form capsules. To study the effects of lamellolysin on Drosophila lamellocytes in vitro, a giant strain of D. melanogaster was constructed to yield hemolymph with an abundance of lamellocytes. The effect of lamellolysin on the adhesivity of lamellocytes in vitro was demonstrated when the cells were gently rotated in the culture medium. Under these conditions, the bipolar shape of the affected lamellocytes resembled that of lamellocytes in parasitized hosts. When lamellocytes were exposed to lamellolysin in stationary culture medium, the elongation of the bipolar cells continued until they became threadlike. Lamellocytes fragmented in both stationary and rotating culture medium in the presence of lamellolysin, although loss of cellular material was more pronounced in the latter. This study demonstrates that lamellolysin acts directly and destructively on lamellocytes.
\end{abstract}

Survival of an endoparasitoid within the hemocoel of an insect host depends on its ability to evade or suppress host cellular defense reactions directed against foreign materials. Some parasitoid eggs have surface features that provide passive protection against encapsulation by host hemocytes (Salt, '65; Rotherham, '73; Davies and Vinson, '86). The hemocytes of other host species are inhibited from encapsulating parasitoid eggs by substances that the female parasitoids oviposit along with eggs in the host hemocoel. Among these materials are the viruses in the calyx fluid of Campoletis sonorensis (Edson et al., '81; Davies et al., '87), the accessory gland secretions of Pimpla turionella (Osman, '78); the venom and calyx fluid of Apanteles glomeratus (Wago and Kitano, '85; Kitano, '82), and the factor within the accessory gland reservoir of Leptopilina heterotoma that selectively destroys the capsule-forming hemocytes of Drosophila melanogaster (R. Rizki and T. Rizki, '84).

The hemocytes of $D$. melanogaster larvae that form capsules are discoidal cells called lamellocytes (Rizki, '57; Rizki and Rizki, '79). Within several hours after an $L$. heterotoma female oviposits in a Drosophila larva, the lamellocytes assume a bipolar morphology and lose their adhesiveness (R. Rizki and T. Rizki, '84). The source of the wasp factor responsible for the lamellocyte changes was located by injecting various isolated portions of the female reproductive system into Drosophila larvae. When the contents of a reservoir associated with an accessory gland were injected into Drosophila larvae, the effects on the lamellocytes mimicked those found in parasitized larvae. This organ is referred to as the long gland reservoir, and the factor within this reservoir that alters the lamellocytes was called lamellolysin. The nature of lamellolysin is unknown, but its selectivity for lamellocytes is intriguing. There are no apparent effects on other host cells and organs. Equally interesting is the bipolar lamellocyte morphology induced by this factor.

Identification of the factor within the long gland reservoir that destroys Drosophila lamellocytes requires assaying components of the reservoir for activity against lamellocytes. In vivo assay involving injection of samples into Drosophila larvae is tedious and time consuming. There are also other drawbacks of this technique, such as difficulties in quantifying the amount of material injected into a larva and loss of hemolymph following puncture of the cuticle of anaesthetized larvae. An in vitro assay for lamellolysin would avoid these problems. Furthermore, the kinetics of the changing cell shapes induced by lamellolysin would be easier to study in vitro than in vivo.

Two methods for observing the effects of long 
gland reservoir contents on Drosophila lamellocytes in vitro were utilized in this study. In the first method, hemocytes were maintained in stationary culture medium so that the changes in individual lamellocytes induced by the wasp factor could be followed for several days. The second method involved rotation of hemocyte samples so that blood cells swirled gently in the liquid medium. This technique, which was used to evaluate changes in lamellocyte adhesion as well as morphology in the presence of lamellolysin, simulates the circulation of hemolymph in the hemocoel of a Drosophila larva.

\section{MATERIALS AND METHODS}

Since only the lamellocytes are markedly affected by lamellolysin, it is necessary to have hemolymph samples with many cells of this type for analysis. This requirement is met by using the temperature-sensitive melanotic tumor mutant, tu-Sz $z^{t s}$, of Drosophila melanogaster as a source of hemocytes (Rizki and Rizki, '80). Lamellocytes are abundant in melanotic tumor larvae but not in larvae whose cellular defense systems have not been challenged (Rizki, '57). Unfortunately, the number of hemocytes obtained from bleeding a single Drosophila larva is small, so hemolymph must be collected from many $t u-S z^{t s}$ larvae to assay a number of fractions separated from the long gland reservoir in a single experiment. To increase the lamellocyte yield per larva, the $t u-S z^{t s}$ gene was combined with alleles of the giant ( $g t$ ) gene, which increases larval size by $70 \%$ (Lindsley and Grell, '68).

Two alleles of the gt gene were separately combined with the $t u-S z^{t s}$ allele. The genotypes of the stocks are 1) gt $w^{a} t u-S z^{t s}$ and 2) y sc gt $t^{X I I} v^{81 i x} t u$ $S z^{t s} / w^{+} Y$ oे ${ }^{+}$and $C(1) D x y w f / w^{+} Y \uparrow क$ (for explanation of symbols, see Lindsley and Grell, '68). Matings between females of the former stock and males of the latter stock give viable female larvae of the genotype gt $w^{a} t u-S z^{t s} / y s c g t^{X I I} v^{81 i x} t u-S z^{t s}$. The larval life of these $g t$ heterozygotes is more than double that of $t u-S z^{t s}$ larvae, and they continue to accumulate lamellocytes during this extended growth period. These larvae are referred to as giant larvae in this paper. The melanotic tumor phenotype is expressed in the giant larvae when they are grown at $27^{\circ} \mathrm{C}$ just as in $t u-S z^{t s}$ larvae raised at this temperature (Fig. 1). Not only does the volume of hemolymph obtained from the giant larvae exceed that of $t u-S z^{t s}$ larvae but the hemolymph of these larvae contains an abundance of lamellocytes.

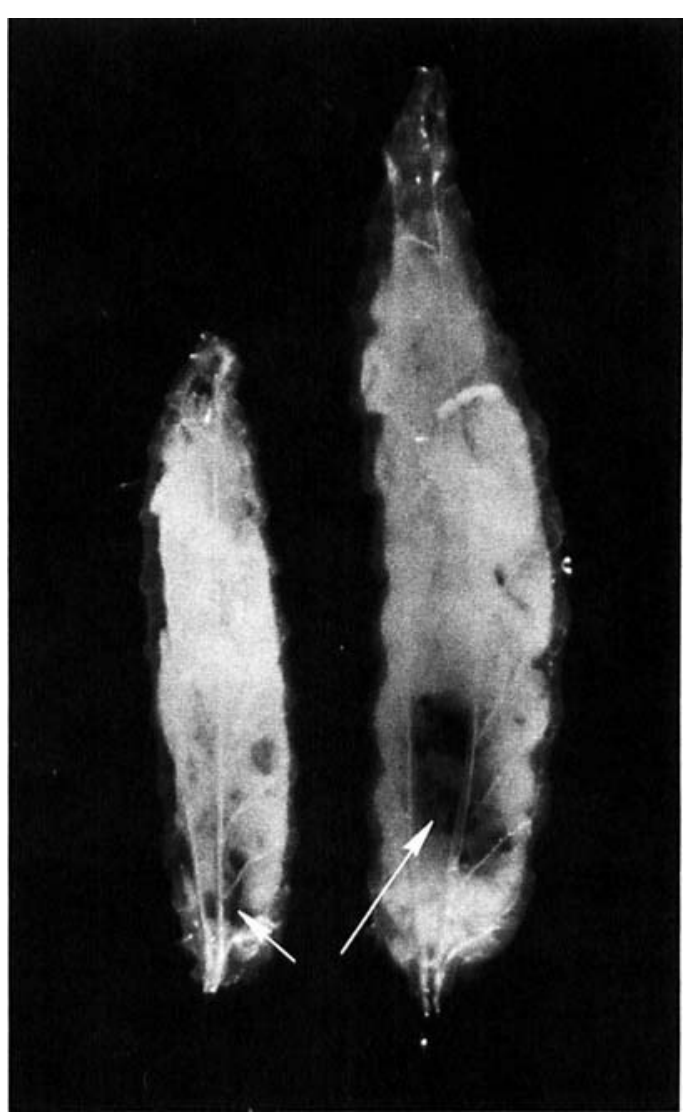

Fig. 1. Comparison of the size of a $t u S z^{t s}$ larva (left) and a giant larva. The arrows point to the melanotic tumors of the caudal fat bodies.

Giant larvae were raised on cream of wheat/ molasses medium seeded with live Fleischmann's yeast at $27^{\circ} \mathrm{C}$. To set up axenic cultures used in some of the studies, flies were allowed to lay eggs on the surface of cream of wheat medium for 15 $\mathrm{hr}$. The eggs were collected from the medium surface with a fine brush, rinsed twice in distilled $\mathrm{H}_{2} \mathrm{O}$, and dechorionated in $5.25 \%$ sodium hypochlorite (Clorox bleach). After four rinses in distilled $\mathrm{H}_{2} \mathrm{O}$, they were sterilized by immersion in povidone-iodine (Betadine solution) for $1 \mathrm{~min}$, rinsed twice in sterile distilled $\mathrm{Hd} 2 \mathrm{O}$, and transferred to the surface of sterile medium, which consisted of $1 \%$ agar, $5 \%$ glucose, and $5 \%$ brewers yeast.

Hemolymph from 8-10-day-old giant larvae was collected in sterile Schneider's culture medium (GIBCO) supplemented with $15 \%$ fetal calf serum. For some studies hemolymph was collected in sterile phosphate buffer (PB) for Drosophila cells (Seecof, '71). Phenylthiourea $(0.07 \%)$ was added to both solutions to inhibit phenoloxidase activity so the hemolymph samples 
would not darken. Hemocyte samples intended for long-term observation were set up under sterile conditions so that lamellocyte morphology could be examined for several days in the absence of contaminating microorganisms.

Stationary samples were set up in $7 \times 32 \mathrm{~mm}$ Sykes chambers with a $1.5 \mathrm{~mm}$ Sykes-Moore-Oring between the lower and upper coverglasses. Each chamber contained $135 \mu$ l of liquid with hemolymph from one or two giant larvae. The cells in these chambers were examined with an Olympus CK2 inverted microscope and photographed with a $35 \mathrm{~mm}$ camera.

Hemolymph samples that were gently swirled on a rotary shaker are referred to as rotation samples. A sample $(75 \mu \mathrm{l})$ was placed in a siliconized flat-bottom depression slide covered with a round $25 \mathrm{~mm}$ coverglass held in place over a silicone gasket by petroleum jelly. The slides were placed on the table of a rotary shaker making 70 revolutions per minute. Under these conditions, the liquid on the slides swirled gently. This degree of movement was used previously to assess the agglutination of Drosophila cells treated with lectins (Andrews and Rizki, '78), so it was deemed adequate to evaluate the adhesivity of lamellocytes in vitro. The hemocyte samples were transferred to microscope slides for examination by dark field illumination or phase contrast and photographed with a Polaroid-back camera.

Leptopilina heterotoma (Leiden strain) were bred on $t u-S z^{t s}$ larvae. Adult parasitoids were maintained on $50 \%$ honey solution in distilled $\mathrm{H}_{2} \mathrm{O}$ at $18^{\circ} \mathrm{C}$. Female wasps were rinsed with $95 \%$ alcohol, followed by three rinses in sterile distilled $\mathrm{H}_{2} \mathrm{O}$ before they were dissected in PB. Long gland reservoirs were collected in $5 \mu \mathrm{PB}$ and torn with fine forceps to release their whitish contents immediately before it was added to Drosophila hemocyte samples. For each experiment, a control sample inoculated with $5 \mu \mathrm{l} \mathrm{PB}$ was included. The parasitoid females used for studies employing sterile cultures were treated with Betadine solution and rinsed in sterile distilled $\mathrm{H}_{2} \mathrm{O}$ before they were dissected under sterile conditions to isolate reservoirs. The use of trypan blue exclusion to assess lamellocyte viability followed the procedure given by Mishell and Shiigi ('80).

\section{RESULTS}

To assess the effects of long gland reservoir fluid on lamellocytes maintained in quiescent culture medium, aliquots of hemolymph in
Schneider's medium were dispensed to two Sykes chambers under sterile conditions. For each experiment, Leiden reservoir fluid from two to five female wasps was added to one chamber, and the other chamber was set up as the control to observe the morphology of lamellocytes in Schneider's medium. Both hemocyte samples of each experimental group were examined immediately after they were made to confirm that the lamellocytes were normal. Since only one inverted microscope was available for the study, only the chamber containing reservoir fluid was kept on the microscope stage for intermittent observation. The control sample was examined again after the conclusion of the observations on the treated sample, so the same cells in control samples were not observed during the course of a given experiment.

Lamellocyte clumps are common in hemolymph samples from $t u-S z^{t s}$ larvae. Figure $2 \mathrm{~A}$ shows a typical group of lamellocytes in a sample examined 15 min $\left(t_{0: 15}\right)$ after the initial $\left(t_{0}\right)$ introduction of cells and reservoir fluid into the chamber. There was no difference between these cells and cells in the control chamber. At $t_{1: 30}$, there were no apparent changes in the cells in this clump, and the field of observation was moved to photograph isolated lamellocytes. Four lamellocytes with the normal discoidal morphology were photographed at $t_{1: 40}$ (Fig. 2B) and when they had assumed a bipolar shape at $t_{2: 50}$ (Fig. $2 \mathrm{C}$ ). It should be noted that lamellocytes remaining suspended in the liquid medium occasionally rotate even though effort is made not to disturb the microscope stage. The lamellocyte at the bottom of the frame in Figure 2C has floated so that its lateral profile is apparent in the photograph. At $t_{3: 05}$, there were no further signs of elongation of these lamellocytes, and the slide was moved to observe other cells.

The elongation of the tip of a single lamellocyte was followed from $t_{3: 05}$ to $t_{3: 35}$. The rate of extension at one end of this lamellocyte was so rapid that the growth of the tip could be discerned by eye while the cell was being observed (Fig. 3A$\mathrm{H})$. The extension rate of this tip was calculated to be $2 \mu \mathrm{m} / \mathrm{min}$. Rapidly growing cellular extensions were also observed in other samples of lamellocytes exposed to reservoir fluid, but the changes in lamellocyte shape do not always proceed so rapidly. The series of photographs in Figure 3 also illustrates a slight curving of the growing tip. This curving motion may be associated with the growth of the tip itself or may be caused 

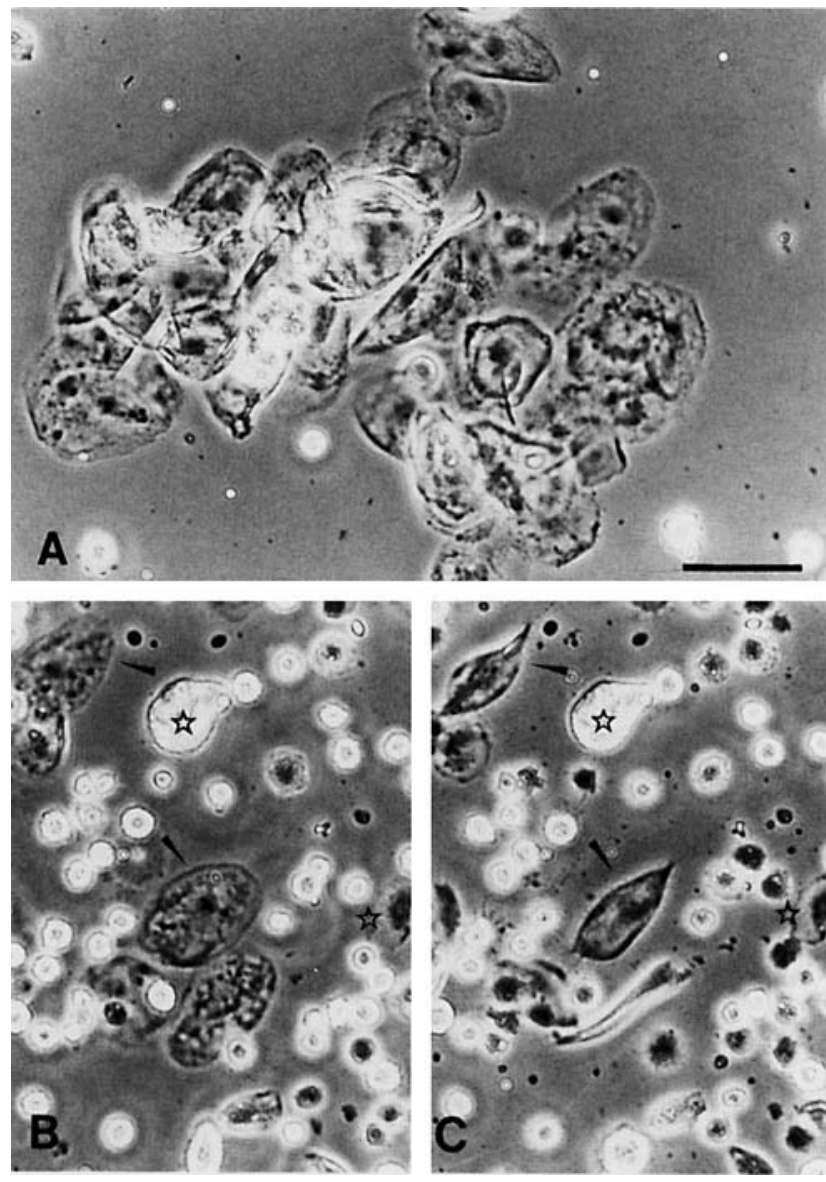

Fig. 2. A: Typical aggregate of lamellocytes in a hemolymph sample in Schneider's medium; stationary culture. B,C: Selected lamellocytes monitored from the same culture as in $\mathrm{A}$ after $1 \mathrm{hr}, 40 \mathrm{~min}$ (B), and after $2 \mathrm{hr}$ and $50 \mathrm{~min}$ (C), of exposure to lamellolysin. Note the shape of the two lamellocytes in the center and the upper left corner (arrowheads) in both photographs. The stars serve as position markers. In $\mathrm{C}$, the change in lamellocyte shape is apparent. The bottom lamellocyte of the central group has floated in the culture medium and is seen in its lateral profile. Bar $=50 \mu \mathrm{m}$.

by movement within the culture medium generated by floating cells. The field in Figure 3 was photographed $17.5 \mathrm{hr}$ later (Fig. 4A), when the lamellocytes were converted into long threads that had no resemblance to their original discoidal morphology. The nuclei remain as spheroidal regions within the extremely elongated cells.

In another experiment, the remnants of elongated lamellocytes were photographed $27 \mathrm{hr}$ after the sample was set up and again at $47 \mathrm{hr}$ when one of the elongated lamellocytes had separated into two pieces (Fig. 4B,C). Small fragments resembling the extending filaments and tips of affected lamellocytes were found in other stationary
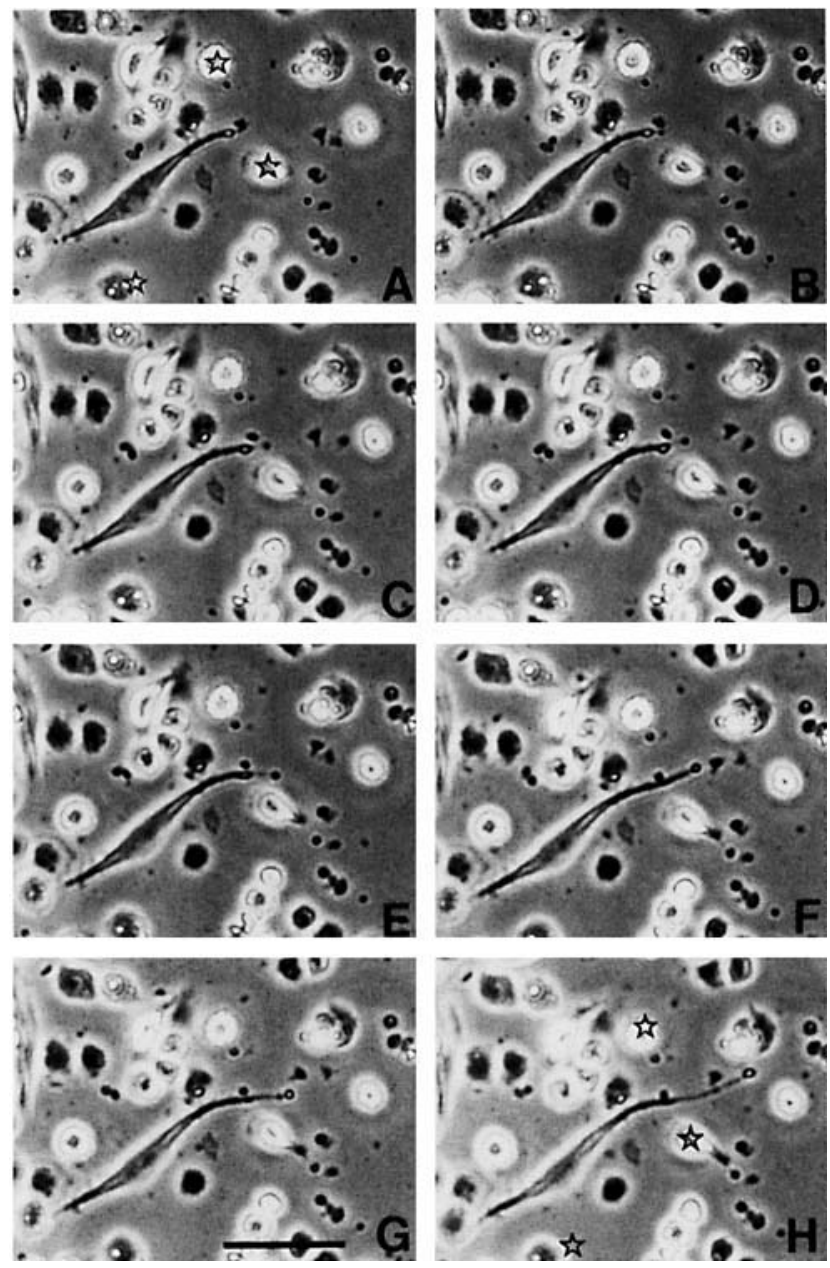

Fig. 3. Series of photographs of an elongating lamellocyte from the same preparation as Figure 2. The stars (in A and $\mathrm{H}$ ) represent reference sites to judge the elongation of the tip of the lamellocyte in $30 \mathrm{~min}$ (from $\mathbf{A}$ through $\mathbf{H}$ ). As the cell elongates, an overall thinning of the cell body is apparent. The elongating tip to the right has a small, bright contrasting bleb. Such blebs are common in the elongating cells. Bar $=50$ $\mu \mathrm{m}$.

samples examined on the second and third days.

Lamellocytes in the absence of reservoir fluid did not undergo elongation. Sterile control samples were photographed shortly after preparation and at the conclusion of each experiment. By the second day in vitro, the edges of many of the lamellocytes were curved and folded, and the cells remained in clumps.

To study the effects of reservoir fluid concentration on the transformation of lamellocytes, a sample of hemolymph from eight giant larvae was dispensed to four Sykes chambers. Aliquots of reservoir fluid from 30 Leiden females were distributed to the chambers so that one contained the reservoir fluid equivalent (RFE) to that of 16 


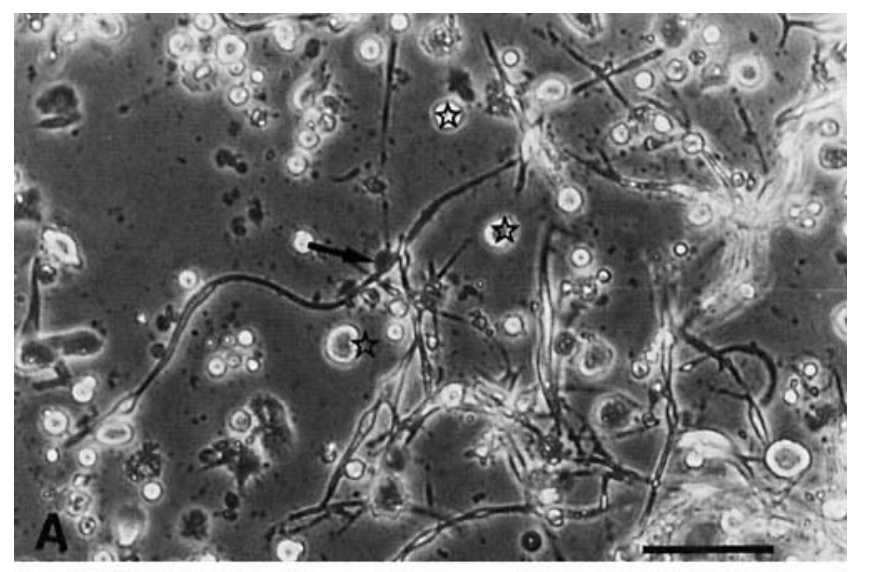

wasps, another chamber received $4 \mathrm{RFE}$, the third had $1 \mathrm{RFE}$, and no reservoir fluid was added to the fourth chamber that served as the control. The total volume of sample in each chamber was $140 \mu \mathrm{l}$, and the series of samples was examined four hours after it was prepared. Two fields with well dispersed lamellocytes were randomly selected and photographed. The normal and affected lamellocytes in each photograph were counted to estimate the percentage of affected cells. The total number of lamellocytes counted in $16 \mathrm{RFE}$ was 94 , in 4 RFE 68 , in 1 RFE 61 , and in the control ( 0 RFE) 40 . The percent of affected lamellocytes in the samples was $100 \%, 96 \%, 75 \%$, and $0 \%$, respectively. The information from this series combined with observations in the above-described studies in which fluid from two reservoirs was added to yield total sample volumes of $135 \mu$ indicates
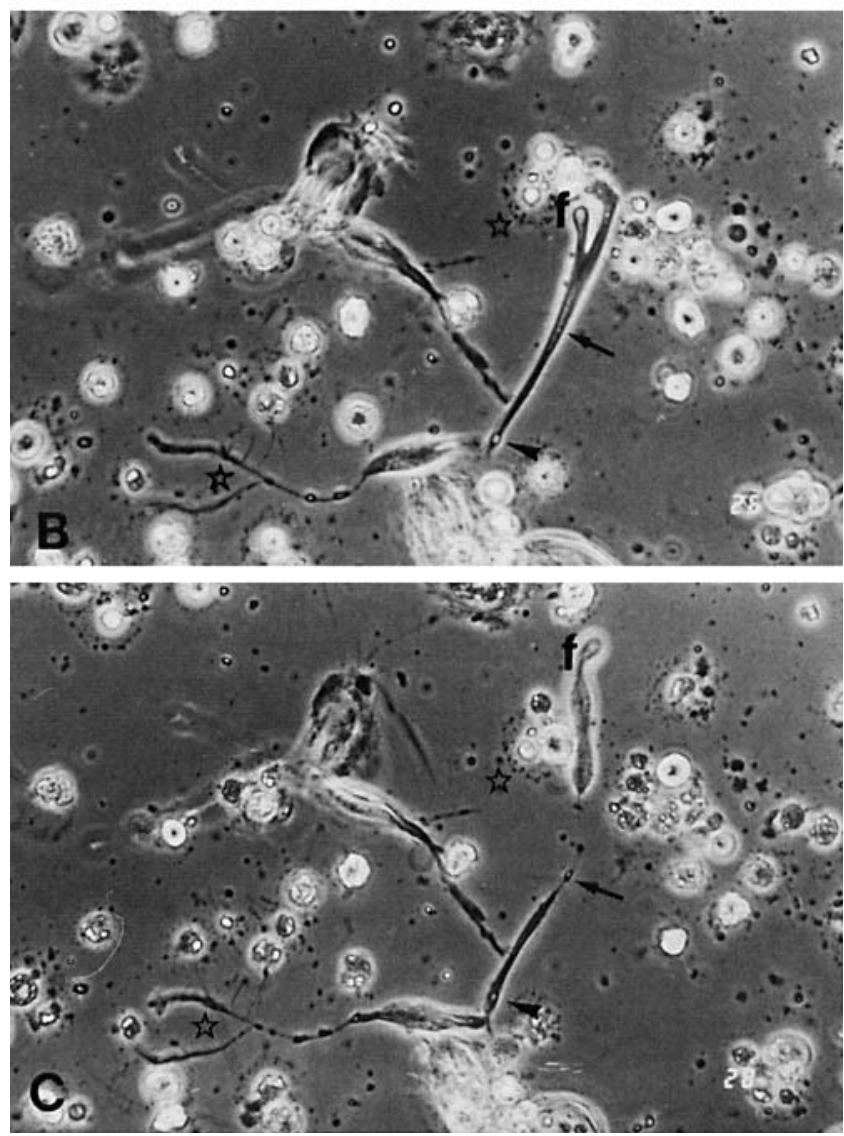

Fig. 4. A: Larger area, showing the same lamellocyte as in Figure 3 photographed $17.5 \mathrm{hr}$ later, when this cell and other lamellocytes in the field had converted to threads. The arrow points to the nucleus of the lamellocyte. $\mathrm{Bar}=50 \mu \mathrm{m}$. B,C: Two photographs of the same elongating lamellocyte from another stationary culture containing larnellolysin photographed at $27 \mathrm{hr}(\mathrm{B})$ and $47 \mathrm{hr}(\mathrm{C})$. The arrowhead and arrow indicate the same parts of the cell in B and C. The forked (f) region in $B$ has broken near the point of the arrow and rotated in $\mathrm{C}$. The stars are position markers. lamellocyte changes under these conditions of incubation in vitro.

Not only was there a positive quantitative correlation between RFE dosage and affected lamellocyte frequency, but the quality of effect was more pronounced at higher dosage. In hemolymph samples from parasitized larvae and in hemolymph samples treated with reservoir fluid in vitro, an occasional bipolar lamellocyte has more than a single elongating filament at one of the poles. This phenomenon was more frequent at the higher RFEs, so it was quantified by counting the number of filaments at the poles of affected cells. In the 16 RFE sample, $17 \%$ of the affected lamellocytes had one filament at one pole and two or more (up to five) filaments at the opposite pole. At 4 RFE, $5 \%$ of the affected lamellocytes had two filaments at one pole and one filament at the other pole. No multifilamentous poles were found in the photographs of the $1 \mathrm{RFE}$ sample.

The plasmatocytes of Drosophila larvae are round, phagocytic cells (Rizki, '57; T. Rizki and R. Rizki, '84) which we found not to be detectably altered by parasitization (R. Rizki and T. Rizki, '84). Plasmatocytes in reservoir-treated samples looked the same as plasmatocytes in control samples for several hours. In older samples containing reservoir fluid, however, there was variation in morphology among the plasmatocytes that was absent in the control samples. To observe individual plasmatocytes for extended periods, two experiments with paired samples, one with and one without reservoir fluid, were set up. In each experiment, plasmatocytes in both samples were examined and photographed immediately after the 
Sykes chambers were sealed. In the first experiment, photographs of plasmatocytes in the chamber with reservoir fluid were taken several hours later, and the same fields were photographed the following day when plasmatocytes in the control sample were also examined. In the second experiment, the Sykes chamber containing the control sample was kept on the microscope stage so that the same fields of unperturbed plasmatocytes were photographed on the first and second days of the experiment. Within 5-15 min after the samples were prepared, some plasmatocytes in both control and treated samples spread and flattened against the glass surface of the Sykes chambers. When the lamellocytes in the treated sample showed early signs of elongation at $2 \mathrm{hr}$, many of the plasmatocytes had spread on the glass surface. At $18 \mathrm{hr}$ when the lamellocytes had extended to a thread-like shape and the surrounding medium contained cellular debris and fragments, some of the flattened plasmatocytes appeared crinkled and had dark and light contrasting areas, producing a daisy-like appearance. The borders of these cells were still smooth, however, and some of the plasmatocytes looked to be unchanged from the previous day. A few plasmatocytes were not spread against the glass surface in the older sample. Plasmatocytes in the control sample photographed on the second day looked the same as they had the previous day. The Schneider's medium in these samples remained clear of debris.

Rotation samples were set up in pairs such that one sample contained reservoir fluid and the other sample did not. In the first experiment, the lamellocytes that had been in Schneider's medium on the rotary shaker for $2.5 \mathrm{hr}$ appeared more vacuolated and folded than fresh lamellocytes in either Schneider's medium or PB. Therefore, the samples removed from the rotary shaker were transferred to $0.5 \mathrm{ml}$ Eppendorf tubes and centrifuged for $1 \mathrm{~min}$ to pellet the cells. The Schneider's medium was removed from the tubes and the cells were gently dispersed in $75 \mu \mathrm{l}$ PB. The cells in PB were returned to depression slides on the rotary shaker for an additional $7 \mathrm{hr}$ before they were reexamined. The morphology of the lamellocytes in these samples that had been in vitro for more than $3.5 \mathrm{hr}$ was similar to that of lamellocytes in fresh samples. In this same experiment, hemocyte samples in Schneider's medium containing the fluid from eight Leiden reservoirs were also transferred to $\mathrm{PB}$ after $2.5 \mathrm{hr}$ as described above. When examined at $3.5 \mathrm{hr}$, the lamellocytes in these samples were bipolar similar to those found in stationary samples at 3-4 hr and in hosts parasitized by Leiden females ( $R$. Rizki and T. Rizki, '84). Therefore, the protocol of an initial $2.5 \mathrm{hr}$ incubation in Schneider's medium followed by a subsequent $1 \mathrm{hr}$ period in $\mathrm{PB}$ was adopted for short-term $(<4 \mathrm{hr})$ rotation samples.

Most of the lamellocytes in control samples on the rotary shaker for $3.5 \mathrm{hr}$ were clumped, indicating that the cells had retained their ability to adhere to one another in vitro (Fig. 5A). Individual lamellocytes in these samples had the normal discoidal morphology (Fig. 5C). The lamellocytes exposed to Leiden reservoir fluid under these same conditions did not adhere to each other and had the elongated morphology typical of affected lamellocytes from larvae parasitized by Leiden wasps (Fig. 5B,D). (R. Rizki and T. Rizki, '84).

To determine the number of reservoirs required to induce lamellocyte changes using the rotation sample procedure, the contents of 16 reservoirs were collected in $20 \mu \mathrm{l}$ of PB. A $10 \mu$ l aliquot from the sample, i.e., $8 \mathrm{RFE}$, was tested for activity, and the remaining $10 \mu \mathrm{l}$ was used to prepare a dilution series containing 4, 2, 1 , and $0.5 \mathrm{RFE}$. The lamellocytes in samples incubated with 4 and $8 \mathrm{RFE}$ were elongated as illustrated in Figure 5D. With 2 RFE, some lamellocytes were elongated, but others appeared normal. Fewer elongated lamellocytes were found in samples with $1 \mathrm{RFE}$, and the lamellocytes in the final dilution of the series (0.5 RFE) appeared normal with an occasional distorted cell. The total volume in a rotation sample was $75 \mu \mathrm{l}$, so the quantity of reservoir fluid required to produce noticeable lamellocyte effects under these conditions was similar to that found for stationary cultures.

Aliquots of a hemolymph sample were used for a series of four cultures to examine the effects of maintaining lamellocytes in constantly moving medium for $24 \mathrm{hr}$. Two cultures contained aliquots of reservoir fluid and two did not. One control sample and one sample with reservoir fluid were removed from the rotary shaker for examination at $4 \mathrm{hr}$, and the remaining two samples were kept on the rotary shaker for an additional $20 \mathrm{hr}$ before they were examined. At 4 and $24 \mathrm{hr}$, the lamellocytes in the control samples were discoidal and clumped. The lamellocytes in the sample with reservoir fluid for $4 \mathrm{hr}$ were dispersed and elongated, as shown in Figure 5B,D. The lamellocytes that had been exposed to reservoir fluid in rotating medium for $24 \mathrm{hr}$ were also dis- 

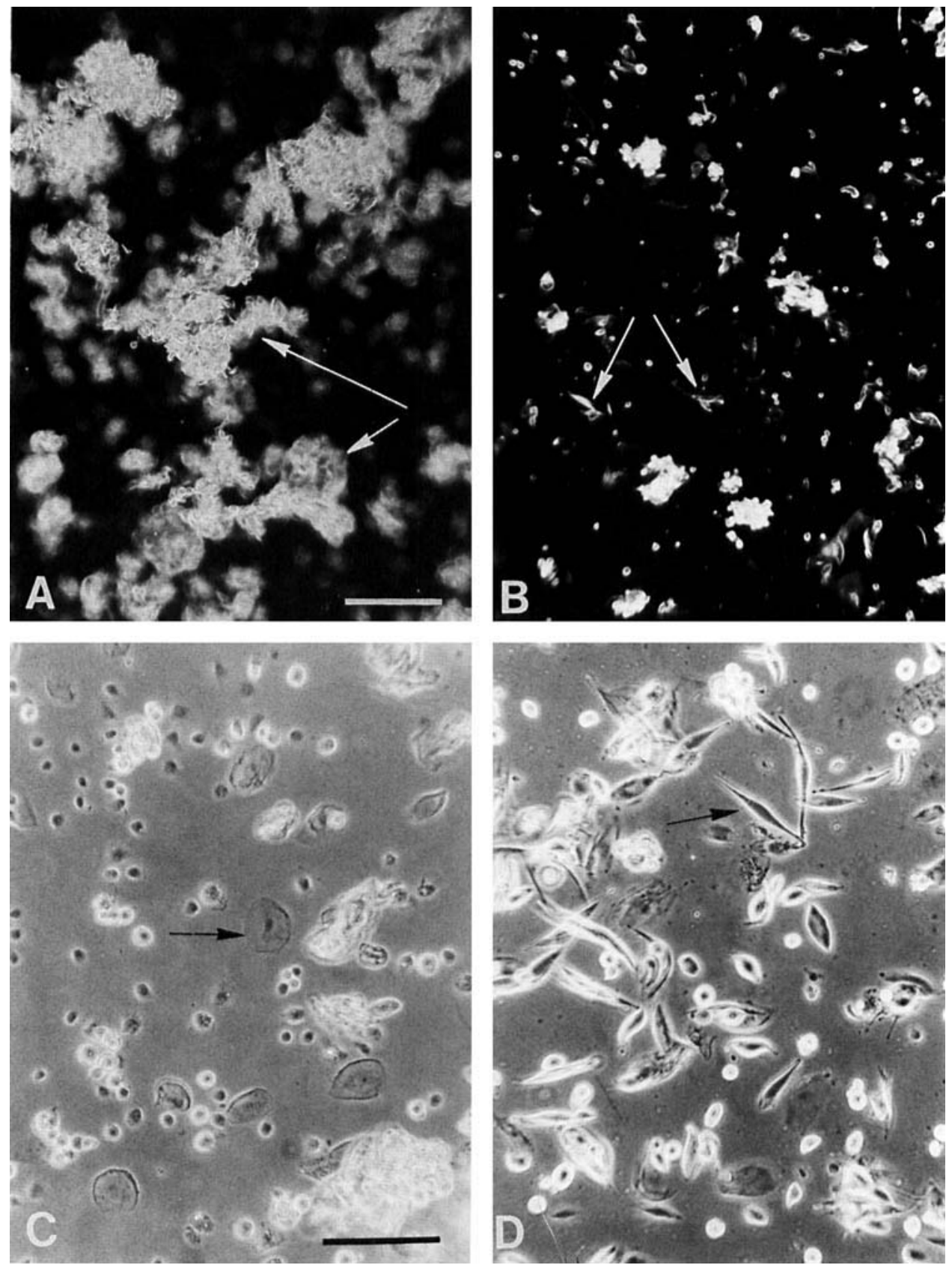

Fig. 5. Hemocytes removed from cultures after $3.5 \mathrm{hr}$ on the rotary shaker. A: Photograph at low magnification showing floating aggregates of lamellocytes (arrows) in a control culture. The discoidal shape of the normal lamellocytes (arrow) in this sample is shown at higher magnification in C. B: Absence of lamellocyte aggregates in the presence of lamel-

persed and elongated. The bipolar ends of many of the latter were knobbed or blunt, and the ratio of nucleus to cytoplasm appeared to be larger than the ratio of nucleus to cytoplasm in the lamellocytes examined at $4 \mathrm{hr}$. Another pronounced difference between control samples and samples

lolysin. The arrows point to individual elongated lamellocytes. The small clumps are plasmatocytes. D: Cells from the lamellolysin-treated sample in B at higher magnification; the morphology of the elongated cells can be seen (arrow). A,B: dark field illumination, bar $=200 \mu \mathrm{m}$. C,D; phase contrast, bar $=100 \mu \mathrm{m}$.

with reservoir fluid was the amount of debris and small particles in the latter. Presumably these were materials extruded from the cells exposed to lamellolysin.

For two experiments, hemolymph was collected in PB rather than in Schneider's medium. These 
samples were intended for short-term $(6 \mathrm{hr})$ incubation only because the buffer does not contain essential cell nutrients present in Schneider's medium. They were also planned for use in cell viability studies with trypan blue to avoid the possibility of trypan blue staining the serum in Schneider's medium. Lamellocyte changes resulting from lamellolysin occurred as rapidly in this buffer as in Schneider's medium, and the lamellocytes in PB control samples looked normal. Trypan blue was added to control and treated cultures in PB at $4 \mathrm{hr}$. Dye was not detected in the lamellocytes, but the nucleoli of the plasmatocytes in both control and treated samples were lightly colored about $5 \mathrm{~min}$ after addition of the dye. Trypan blue was also added to control and reservoir-treated hemocytes that had been in Schneider's medium for $4 \mathrm{hr}$. The cells in Schneider's medium responded to the dye the same as the cells in PB. Stationary hemocyte samples that had been in Schneider's medium for $24 \mathrm{hr}$ and contained threaded lamellocytes were also treated with trypan blue. The threaded lamellocytes in the samples were not colored with the dye. Materials that appeared to be cell fragments and a few plasmatocytes picked up the dye. It was surprising that the dye did not diffuse into the threaded lamellocytes, which we presumed to be dead. To verify that trypan blue would enter dead lamellocytes, the cells in 24-hr-old cultures were heat killed by placing the chambers containing cells in a $60^{\circ} \mathrm{C}$ oven for 10 min prior to addition of trypan blue. All cellular material from the heattreated chambers, including the threaded lamellocytes, immediately was colored blue.

\section{DISCUSSION}

The present study confirms that the long gland reservoir of the $L$. heterotoma female contains an antilamellocyte factor (R. Rizki and T. Rizki, '84). It is clear that this factor acts directly on lamellocytes, since the in vitro system contains only hemocytes and wasp reservoir fluid. Furthermore, the effects of lamellolysin on lamellocytes in vitro parallel the changes seen in these cells in vivo following parasitization by wasps or by injection of reservoir fluid into Drosophila larvae. In all three cases, the lamellocytes assume a bipolar shape and lose their ability to adhere to each other. The loss of adhesivity destroys the functional capability of the lamellocytes to adhere to foreign surfaces and to engage in encapsulation of foreign objects. The absence of functional lamellocytes in a Drosophila host ensures that parasitoid eggs remain unencapsulated and free to develop.

We presume that the lamellolysin affect on the shape of the lamellocyte is related to the loss of lamellocyte adhesiveness. However, until the nature of lamellolysin is established and its molecular action on lamellocytes is understood, there is no evidence excluding the possibility that lamellolysin has more than a single target in the lamellocyte. Examination of intracellular changes involved in the morphological transformation of the lamellocytes following exposure to lamellolysin has been limited to the disposition of the cytoskeleton (R. Rizki and T. Rizki, '84). Rearrangement of microtubule materials occurs during the modification from a disk to a bipolar shape, but this is expected since the disposition of cytoskeleton dictates cell shape. If the plasticity of the cell surface is lost in the stretching lamellocyte, then adhesive properties of the cells may be modified as a result of the elongation process induced by lamellolysin.

Exposure to higher concentrations of reservoir fluid not only increased the number of affected lamellocytes but also resulted in an increase in lamellocytes with multiple tips extending from the poles. The latter observation suggests that there are multiple targets or receptor sites for lamellolysin on the cell surface. More intensive study of the relationship between lamellolysin concentration and cell morphology is required as well as detailed mapping of the points from which cytoplasmic projections emanate in affected cells. Why the affected cells generally assume a bipolar shape also requires explanation. The reorientation of microtubule components occurring as a consequence of lamellolysin activity must be directed along a single or restricted axis to result in a bipolar cell.

The distortion of plasmatocytes in overnight cultures containing reservoir fluid may be a delayed effect of exposure to lamellolysin or a secondary effect resulting from the release of lytic factors from the distintegrating lamellocytes in the older cultures. Distinction between these alternatives should be possible by using hemolymph sample devoid of lamellocytes. No diferences between plasmatocytes in control cultures and in cultures with reservoir fluid were detectable when effects on lamellocytes were apparent in the latter on the first day of treatment. Plasmatocytes in both samples spread against the glass surface within $15 \mathrm{~min}$ and the morphology of the flattened cells was the same in the presence or absence of lamellolysin. Plasmatocytes of 
parasitized larvae also appeared normal (R. Rizki and T. Rizki, '84). The susceptibility of lamellocytes to lamellolysin may relate to some unique feature of this cell type.

The effect of wasp reservoir fluid on lamellocyte adhesivity can be assessed in vitro when the culture medium is subjected to movement that allows contact between the cells. Under these conditions, the shape of the elongated lamellocytes closely resembles that of lamellocytes from parasitized hosts (R. Rizki and T. Rizki, '84). The affected cells generally have a single fine tip or a blunt knob at each pole. The accumulation of cellular fragments in the culture medium also mimics the accumulation of cellular debris found in the hemolymph of parasitized Drosophila larvae. If portions of cytoplasm are shed from the lamellocytes in circulating hemolymph or culture medium, then the ratio of nucleus to cytoplasm should increase. In older rotation samples, the larger volume of the cell occupied by the nucleus was readily apparent. Even under quiescent conditions, which apparently do not favor fragmentation, instances of this phenomenon were recorded. Thus there is no doubt that lamellolysin causes destruction of Drosophila lamellocytes. This orderly destructive process must depend on the vitality of the cells, since the affected lamellocytes exclude trypan blue. Transformation of the lamellocytes from discoidal cells to thread-like cells was observed only when the cells remained undisturbed in vitro. The circulation of hemocytes in the hemolymph of parasitized hosts must be sufficient to buffet the bulk of the elongating lamellocytes such that their elongating tips break free.

\section{ACKNOWLEDGMENTS}

This work was supported by NSF grant DCB8517807 to R.M.R. and NIH grant GM-37025 to T.M.R. We thank Pearl Johnson for typing the manuscript.

\section{LITERATURE CITED}

Andrews, C.A., and T.M. Rizki (1978) Studies on lectininduced agglutination of Drosophila embryonic cell lines. J. Insect Physiol., 24:9-12.
Davies, D.H., and S.B. Vinson (1986) Passive evasion by eggs of braconid parasitoid Cardiochiles nigriceps of encapsulation in vitro by haemocytes of host Heliothis virescens. Possible role for fibrous layer in immunity. J. Insect Physiol., 32:1003-1010.

Davies, D.H., M.R. Strand, and S.B. Vinson (1987) Changes in differential haemocyte count and in vitro behavior of plasmatocytes from host Heliothis virescens caused by Campoletis sonorensis polydnavirus. J. Insect Physiol., 33:143153.

Edson, K.M., S.B. Vinson, D.B. Stoltz, and M.D. Summers (1981) Virus in a parasitoid wasp: Suppression of the cellular immune response in the parasitoid's host. Science, 211:582-583.

Kitano, H. (1982) Effect of the venom in the gregarious parasitoid Apanteles glomeratus on its hemocytic encapsulation by the host, Pieris. J. Invertebr. Pathol., 40:61-67.

Lindsley, D.L., and E.H. Grell (1968) Genetic Variations of Drosophila melanogaster. Carnegie Inst. Wash. Publ. 627, p. 472 .

Mishell, B.B., and S.M. Shiigi (1980) Selected Methods in Cellular Immunology. W.H. Freeman and Company, San Francisco, p. 486.

Osman, S.E. (1978) Die Wirkung der Sekrete der weiblichen Genitalanhangsdrüsen von Pimpla turionellae L. (Hym., Ichneumonide) auf die Hämocyten und die Einkapselungsreaktion von Wirtspuppen. Z. Parasitenkd., 57:89100.

Rizki, R.M., and T.M. Rizki (1979) Cell interactions in the differentiation of a melanotic tumor in Drosophila. Differentiation, $12: 167-178$.

Rizki, R.M. and T.M. Rizki (1984) Selective destruction of a host blood cell type by a parasitoid wasp. Proc. Natl. Acad. Sci. USA, 81:6154-6158.

Rizki, T.M. (1957) Tumor formation in relation to metamorphosis in Drosophila melanogaster. J. Morphol., 100:459472.

Rizki, T.M., and R.M. Rizki (1980) Developmental analysis of a temperature-sensitive melanotic tumor mutant in Drosophila melanogaster. Wilhelm Rouxs Arch., 189:197206.

Rizki, T.M., and R.M. Rizki (1984) The cellular defense system of Drosophila melanogaster. In: Insect Ultrastructure, Vol., 2. R.C. King and H. Akai, eds. Plenum Publishing Corp., New York.

Rotheram, S. (1973) The surface of the egg of a parasitic insect II. The ultrastructure of the particulate coat on the egg of Nemeritis. Proc. R. Soc. London [Biol.], 183:195-204.

Salt, G. (1965) Experimental studies in insect parasitism XIII. The haemocytic reaction of a caterpillar to eggs of its habitual parasite. Proc. R. Soc. London [Biol.], 162:303318.

Seecof, R.L. (1971) Phosphate-buffered saline for Drosophila. Drosophila Inform. Serv., 46:113.

Wago, H., and H. Kitano (1985) Effects of the venom from Apanteles glomeratus on the hemocytes and hemolymph of Pieris rapae crucivora. Appl. Entomol. Zool., 20:103-110. 